\title{
With regard to the article "Acute abdomen in patients with SARS-CoV-2 infection or co-infection"
}

\author{
Barbara Seeliger $^{1,2}$ (D) Patrick Pessaux ${ }^{1,2}($ D \\ Received: 14 September 2020 / Accepted: 15 September 2020 / Published online: 22 September 2020 \\ (C) Springer-Verlag GmbH Germany, part of Springer Nature 2020
}

In order to ensure timely publication of our article "Acute abdomen in patients with SARS-CoV-2 infection or co-infection" [1], the reported follow-up was a minimum of 4 weeks for all patients who underwent emergency abdominal surgery during their COVID-19 disease. It is of importance to address the long-term outcome of surgical procedures, and particularly to determine if patients with an acute abdomen have an increased mortality risk due to a SARS-CoV-2 infection. It was hypothesized that elective surgical procedures during the incubation period lead to an exacerbation of COVID-19 disease [2]. In the COVIDSurg Collaborative study we participated in, a first analysis of 1128 patients with perioperative SARSCoV-2 infection showed a $19.1 \%$ postsurgical mortality rate in elective surgery, even surpassed by a $26.0 \%$ mortality rate in emergency surgery during a 30-day follow-up [3]. We would herewith like to complement a 3-month follow-up for our case series.

Among our patients, two scenarios had been identified according to the time point of their emergency surgical procedure: (A) patients who were admitted to the hospital for an acute surgical condition with a concomitant diagnosis of COVID-19 and (B) patients with severe COVID-19 developing acute abdominal pathologies during their hospital stay.

A total of three patients had died during initial follow-up (A5: POD 1; B1: POD 11; B4: POD 48). After a mean followup of $132 \pm 26$ days (median 131, range 94-163), no further patients have died during long-term follow-up.

Patrick Pessaux

patrick.pessaux@chru-strasbourg.fr

1 IHU-Strasbourg, Institute of Image-Guided Surgery, Strasbourg, France

2 Department of Visceral and Digestive Surgery, Nouvel Hopital Civil, 1 place de l'hôpital, 67091 Strasbourg, France
Patients in scenario A performed better in terms of pulmonary and overall outcomes. By now, there are no more pulmonary or digestive residual symptoms in these patients (A1-4, A7).

One patient (A1, age 31) was re-hospitalized for drainage of a pelvic collection as a result of a rectal stump insufficiency after a Hartmann's procedure for colonic perforation due to obstruction. Relevant medical history includes LennoxGastaut syndrome with associated psychomotor delay. He was re-hospitalized (POD 44) for a urinary tract infection with prostatitis and pyelitis, treated antibiotically, and repeated pelvic collection drainage. His nasopharyngeal swab was still SARS-CoV-2 positive on postoperative day (POD) 68, the first negative swab was obtained on POD 79. Currently, the patient has no more digestive or pulmonary symptoms (POD 159), but needs physiotherapy to regain previous musculoskeletal forces (global weakness in seated and standing positions).

Patients with an incarcerated femoral hernia (A2, age 81), incarcerated incisional hernia (A3, age 70), appendicitis (A4, age 71), and perforated sigmoid diverticulitis undergoing Hartmann's procedure (A7, age 80) recovered without any complications and were not considerably impaired due to COVID-19 coinfection. A homeless patient (A6, age 44, liver stab wound) not responding to phone calls after discharge was lost to follow-up.

In scenario B, only two patients were discharged home initially.

Patient B2 (age 28), who underwent small-bowel resection for ischemia due to portal and mesenteric venous thrombosis (PMVT) [4], was scheduled for re-hospitalization for reestablishment of digestive continuity on POD 69 (takedown of double-barrel ileostomy and creation of an ileo-ileal anastomosis). The postoperative course was uncomplicated. The patient is now asymptomatic, including absence of digestive symptoms from the previous PMVT. The PMVT was the initial manifestation of a subsequently diagnosed JAK2 V617F mutated essential thrombocythemia, currently well 
controlled under hydroxycarbamide treatment. PMVT is a rare disorder, but a familiar presenting complication of previously undiagnosed myeloproliferative diseases, particularly in young patients [5]. Consequently, the patient's prothrombotic state can be attributed not only to her COVID-19 infection but also to the essential thrombocythemia (SWAB positive POD 4, negative POD 11 and all following ones until POD 88).

Patient B5 was hospitalized for COVID-19 disease with an acute respiratory distress syndrome (ARDS). She had an acute renal insufficiency 13 days after admission, and computed tomography showed a voluminous hematoma of the right psoas muscle compressing the right ureter and vena cava with active bleeding. Bleeding persisted after embolization of epigastric and lumbar arteries (L1-L3, L4), and a second embolization (L1-L4) was performed along with a right pyelostomy. Following surgical evacuation of the hematoma, a favorable pulmonary evolution allowed extubation on POD 8. She suffered from neuropathic pain in the right leg, and an electrophysiological exam had shown a femoral nerve damage subsequent to the compressive retroperitoneal hematoma. On POD 40, the patient was transferred to a reeducation center for 1 month. On a digestive and pulmonary level, the patient recovered quickly and is currently asymptomatic. However, she still experiences femoral nerve pain, and her motricity is continuing to recover with ambulatory care twice a week: SWAB positive 6 days preop, negative 3 days preop and following; BAL positive until last one POD 7.

Two further patients needed to stay hospitalized for several months.

Patient B3 (age 56) was hospitalized for severe COVID-19 disease with an ARDS necessitating a total of 59 days of mechanical ventilation. Relevant medical history includes a 20-year disability due to severe insulin-dependent type 2 diabetes with polyneuropathy of the lower limbs and difficulty walking.

After 9 days, he presented a shock of mixed etiology: bilateral pulmonary embolism (presence of circulating lupus anticoagulants), ventilator-associated pneumonia, and mesenteric ischemia. An open small-bowel resection was performed, and a second-look procedure with creation of a double-barrel ileostomy on POD 2 [4]. After failure to wake up after cessation of sedation, a thorough workup showed an acute necrotizing hemorrhagic leukoencephalitis, and a Guillain-Barré syndrome with anti-GM1 antibodies (immunoglobulin and plasmapheresis treatment received). His intensive care unit stay was further complicated and prolonged (in total 139 days) with acute renal insufficiency needing renal dialysis, and multiple septic shocks originating from pressure ulcers and ventilator-associated pneumonias, and critical illness polyneuropathy. Bowel continuity was re-established on POD 73, with creation of a colostomy in order to facilitate healing of a gluteal defect. After failed endoscopic and CT-guided attempts, a surgical gastrostomy was carried out on POD 120 for difficulty swallowing.

Patient B6 (age 70) was hospitalized for COVID-19 disease with an ARDS. She had experienced a hemorrhagic shock from left renal artery bleeding during therapeutic anticoagulation in the presence of circulating lupus anticoagulants. Twenty-five days after admission, she had undergone an open drainage of the hemoperitoneum refractory to embolization. It had been followed by a reduction of incarcerated small bowel within the previous incision, multiple temporary parietal closures during an abdominal compartment syndrome leading to an eventeration, and a cholecystectomy for gangrenous cholecystitis. Her intensive care unit stay and further hospital stay were marked by several septic episodes and the presence of intraabdominal collections. A transfer to a continuing care unit was possible on POD 45, transfer to the internal medicine ward on POD 83, and discharge to a rehabilitation facility on POD 94.

\section{References}

1. Seeliger B, Philouze G, Cherkaoui Z, Felli E, Mutter D, Pessaux P (2020) Acute abdomen in patients with SARS-CoV-2 infection or co-infection. Langenbeck's Arch Surg 405:861-866. https://doi.org/ 10.1007/s00423-020-01948-2

2. Lei S, Jiang F, Su W, Chen C, Chen J, Mei W, Zhan LY, Jia Y, Zhang L, Liu D, Xia ZY, Xia Z (2020) Clinical characteristics and outcomes of patients undergoing surgeries during the incubation period of COVID-19 infection. EClinicalMedicine 21:100331. https:// doi.org/10.1016/j.eclinm.2020.100331

3. COVIDSurg Collaborative (2020) Mortality and pulmonary complications in patients undergoing surgery with perioperative SARSCoV-2 infection: an international cohort study. Lancet 396(10243): 27-38. https://doi.org/10.1016/S0140-6736(20)31182-X

4. Ignat M, Philouze G, Aussenac-Belle L, Faucher V, Collange O, Mutter D, Pessaux P (2020) Small bowel ischemia and SARSCoV-2 infection: an underdiagnosed distinct clinical entity. Surgery 168(1):14-16. https://doi.org/10.1016/j.surg.2020.04.035

5. Colaizzo D, Amitrano L, Tiscia GL, Scenna G, Grandone E, Guardascione MA, Brancaccio V, Margaglione M (2007) The JAK2 V617F mutation frequently occurs in patients with portal and mesenteric venous thrombosis. J Thromb Haemost 5(1):55-61. https://doi.org/10.1111/j.1538-7836.2006.02277.x

Publisher's note Springer Nature remains neutral with regard to jurisdictional claims in published maps and institutional affiliations. 